\title{
Advanced algorithmic model for poly-optimization of biomass fuel production from separate combustible fractions of municipal wastes as a progress in improving energy efficiency of waste utilization
}

\author{
Krzysztof Gaska ${ }^{1,}$, Agnieszka Generowicz ${ }^{2}$, Mykhailo Lobur ${ }^{3}$, Nazariy Jaworski ${ }^{3}$, Józef Ciuła $^{4}$, and Myroslava Vovk $^{5}$ \\ ${ }^{1}$ Faculty of Energy and Environmental Engineering, Silesian University of Technology, Gliwice, Poland \\ ${ }^{2}$ Faculty of Environmental Engineering, Cracow University of Technology, Cracow, Poland \\ ${ }^{3}$ CAD Systems Department, Lviv Polytechnic National University, Lviv, Ukraine \\ ${ }^{4}$ Institute of Technology, State University of Applied Sciences in Nowy Sącz, Nowy Sącz, Poland \\ ${ }^{5}$ Department of Mathematics, Lviv Polytechnic National University, Lviv, Ukraine
}

\begin{abstract}
Usage of generated fuel components from segregated municipal waste groups in combined combustion processes with traditional fuels results in significant savings by partial replacement of fossil fuels and the reduction of environmental inconvenience that arises directly from the migration of waste to the environment. This article develops technological process of fuel components production which is controlled by advanced embedded SCADA control systems, providing monitoring of process parameters, process visualization and implementation, with the help of logic controllers of freely programmable PLC and complex control algorithms (including adaptive, predictive and inferential control algorithms) by implementation of the polymorphic model of fuel components obtaining process multi-stream optimization.
\end{abstract}

\section{Introduction}

Growing demand for heat and electricity used in many industries and utilities leads to a sharp decline in the natural resources of traditional fuels (coal, lignite, oil and natural gas). Excessive exploitation of natural energy sources results, in deterioration of the environment and irreversible climate change. The consequence of energy carriers, products or semifinished products extraction used in various technological processes is the formation of secondary post-technological wastes of different groups. The source of waste (outside the industry) is other entity that produces unsuccessful streams of hazardous waste (medical waste, chemical reagents, including pesticides, etc.). The urban enthalpy of devaluation allows for their reuse after appropriate treatment [1-3].

Due to the use of molded fuel components from separated waste groups in combined combustion processes with conventional fuels one can achieve significant saving thanks to the partial replacement of fossil fuels and the reduction of environmental disadvantages that arise directly from the migration of waste to the environment (soil, atmospheric air, surface water and underground water) [8]. Particular attention should be paid to the possibility of using certain groups of hazardous waste in these processes (pesticides, medical waste). Conducting technological processes by using preformed fuel components in thermally coupled systems should be conditioned by technical criteria, which means that they should not interfere with the main processes, and in the global plan, with the factors of sustainable development. The physical process of preparing a fuel mixture requires careful analysis of the physical and chemical properties of the components, molded fuel components and technological conditions. The technological process of fuel components production is controlled by advanced SCADA-type control systems which provide process parameters monitoring, visualization and implementation with the help of freely programmable PLC logic controllers and complex control algorithms (including adaptive, predictive and inferential control algorithms).

\section{Fuel components production process multi-stream optimization model}

Optimization task of the complex technological process of manufacturing fuel components, formed from separated fractions from combustible municipal waste includes two main threads:

A) optimization of light fraction (calorific value) production consisting of isolation of heavy, nonmetal components from the waste flow (in the optical separator). They have a high calorific value of $\mathrm{Wd}$ exceeding $40 \mathrm{MJ} / \mathrm{kg}$ in the case of $\mathrm{PE}$ (mainly PE, PET, PP plastics). It can be done by using an improved algorithm for forecasting MPC [4]

Corresponding author: krzysztof.gaska@polsl.pl 
control (based on the modified MC SVM (MultiClass Vector Support Machine) algorithm) implemented in the control layer of the SCADA optimization hierarchical control system;

B) optimization of the hybrid fuel components obtaining process which consists in mixing of light fraction (obtained as a result of optimization of the optical separation process - A thread) with fossil fuels that corresponds to the target function, i.e. maximization under restrictions that are imposed on alternatives of optimization problem solution and parameters of implemented technological processes (rotary kilns, boilers, fluidized-bed boilers, etc.). The process optimization is carried out with the help of a modified simplex algorithm.

The choice of mechanical processes and their parameters, as well as the relationship between processes, is based on the knowledge of the morphological composition, the frequency and amount of waste delivered, as well as the concept of further processing of the recovered fractions. Physical properties such as humidity (mechanical sorting is the best for dry waste) and the density of waste are irrelevant. Depending on these parameters, the process line will take another form. These settings may differ not only by the devices used in them but also from the point of view of their number and location in the technological process. Only proper selection of the dividing line separate elements guarantees achievement of the predicted recovery rates of fraction from mixed municipal waste. In this regard, knowledge of the processes used to purchase individual waste fractions is crucial for the development and exploitation of waste segregation lines [5-7].

All elements of the system are connected with a control system based on a complex algorithm defined for a specific fraction of waste. In the context of ensuring the proper conduct of the process, the molded fuel production plant should be considered as a complex, non-linear, multidimensional control object.

The integrated multithreaded process control algorithm, embedded in a programmable logical PLC controller (controlled by the SCADA system), performs the task of solving the optimization problem defined in the form of a support vector machine model (SVM) of non-metallic fraction components (preferably ballistic and optical division), which reproduces the structures of predefined classes - subsets generated by the greedy algorithm of the Greedy Set Cover approximation containing fraction components described by a set of attributes (including physical and chemical properties, fuel properties, emissions, texture, structure, spectroscopic spectra).

Each subgroup is the fuel class component of the fraction composition. It is formed by the optimization effect using Greedy Set Cover approximation algorithm, which maximizes the target function (Wd-value of the heating) when performing the technological constraints imposed on the linear programming tasks (humidity, fraction of chlorine, heavy metals). Reference classes are described by a set of attributes (texture, structure, spectrum) which allow the classification algorithm of the lower level of classification management to be classified using the SVM network model (MC SVM) based on a set of functions assigned to predefined classes represented by disjoint subsets of coverage by the Greedy Set Cover algorithm, and finally verify that the identified component/fraction belongs to the template class on the incident matrix.

The optimization of lightweight fraction production process is implemented in the control layer of the SCADA hierarchical control system (fig. 1,2).

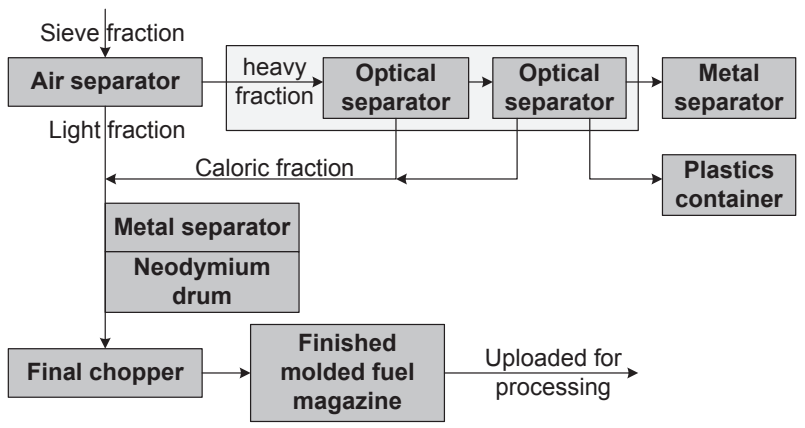

Fig. 1. Block diagram of ballistic and optical separation processes separated from the technological line.

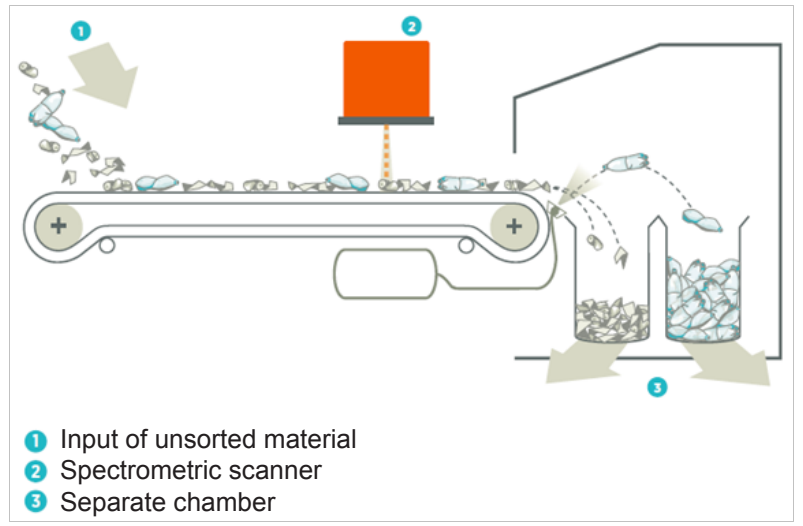

Fig. 2. Optical separator.

\section{Mathematical formulations}

Output signals of the preprocessor in the form of the following components of the Fourier descriptors, after the conversion, providing the invariant to scale, rotation and displacement, are input signals for the multilayer neural network, functioning as a pattern recognition system and simultaneously classification, that is, assigning a template to the corresponding class. The number of network input nodes is equal to the number of Fourier descriptors that are considered in the classification. Given that, each source neuron is a single class, their number is also constant and equals many classes. The classifier learns on a set of training data representing sequential classes of recognizable images (detecting on the basis of spectra, texture, contour, structure). In recovery mode, the classified template after passing all the phases of the preprocessor enters the network input and stimulates the output neuron that corresponds to this class.

At the stage of patterns recognition, through their noises, the source signals of the network neurons can 
accept continuous values from the interval $[0 ; 1]$ instead of the expected binary values, and the first corresponds to the recognized class.

In the classification question, classificatory MC SVM is based on a one-way neural network that implements various types of activation functions, including linear, polynomial, radial and sigmoid. The purpose of the classification is to maximize the separation limit between the two different classes described in the set of pairs $\left(x_{i}, d_{i}\right)$, where $x_{i}$ is the input vector and $d_{i}$ is a given point (for two classes it reaches the value of 1 for class 1 or -1 for class 2). Assuming the linear separation of both classes, the equation dividing the two classes can be preserved in the form:

$$
\begin{gathered}
g(x)=w^{T} x+b=0, \\
w=\left[w_{1}, w_{2}, \ldots, w_{N}\right]^{T}, \\
x=\left[x_{1}, x_{2}, \ldots, x_{N}\right]^{T} .
\end{gathered}
$$

In this equation, taking $N$ inputs, the weight vector $w$ is $N$-dimensional; weight $b$ is polarization. Solutions of the equations that determine the class affiliation is:

$$
\begin{gathered}
w^{T} x_{i}+b>0 ? d_{i}=, \\
w^{T} x_{i}+b<0 ? d_{i}=-,
\end{gathered}
$$

or after the transformation:

$$
\begin{gathered}
w^{T} x_{i}+b \geq 1 ? d_{i}=, \\
w^{T} x_{i}+b \leq-1 ? d_{i}=-.
\end{gathered}
$$

If a pair of points $\left(x_{i}, d_{i}\right)$ corresponds to the above equation with the equals sign, then the vector $x_{i}=x_{s v}$ creates the so-called support vector. They are those data points that are closest to the optimal hyperplane and are the most difficult to classify.

The problem of studying SVM linear networks, i.e. the choice of weights of synaptic connections for linearly separated training data, is to maximize the separation limit (fig. 3). This is a quadratic programming problem with linear weight constraints, which is solved by means of the Lagrange multiplier method by minimizing Lagrange function.

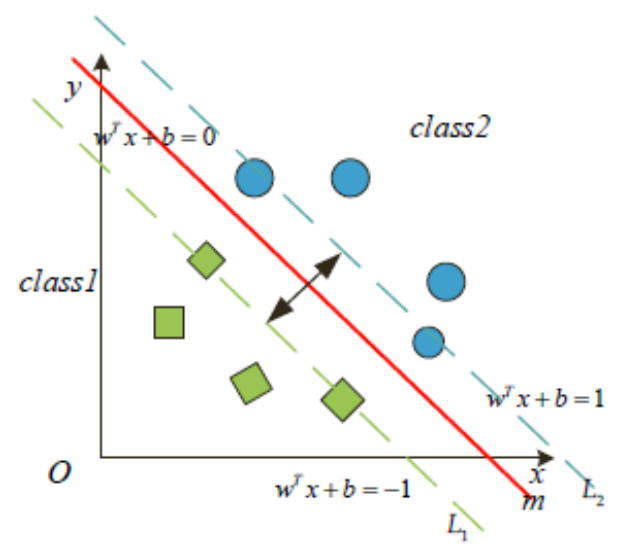

Fig. 3. Interpretation of the margin of separation between two data classes.
Taking into account that the task of training is to maximize the Lagrange function against Lagrange multipliers, the primary problem is converted into a double, which is formulated as follows:

$$
\begin{gathered}
\max \left\{\sum_{i=1}^{p} \alpha_{i}-\frac{1}{2} \sum_{i=1}^{p} \sum_{j=1}^{p} \alpha_{i} \alpha_{j} d_{i} d_{j} x_{i}^{T} x_{j}\right\}, \\
\alpha_{i} \geq 0, \quad \sum_{i=1}^{p} \alpha_{i} d_{i} .
\end{gathered}
$$

The solution of the aforementioned optimization problem to Lagrange multipliers allows one to determine the equation of an optimal hyperplane, which is determined by the $x$-weight vector and the polarization parameter $b$.

When one is solving the problem of classification of indivisible samples, the problem is to determine the optimal hyperplane, which minimizes the probability of classification error on the training set with the maximum possible differentiation. As in the case of linearly separable regularities, the primary problem is reduced to a dual problem, which is formulated in the following way:

$$
\begin{gathered}
\max \left\{\sum_{i=1}^{p} \alpha_{i}-\frac{1}{2} \sum_{i=1}^{p} \sum_{j=1}^{p} \alpha_{i} \alpha_{j} d_{i} d_{j} x_{i}^{T} x_{j}\right\}, \\
0 \leq \alpha_{i} \leq C, \quad \sum_{i=1}^{p} \alpha_{i} d_{i}=0,
\end{gathered}
$$

for $i=1,2, \ldots, p$ and the value of the constant $C$ is accepted by user. Therefore, from the solution of the dual problem one can obtain the expression for weight vector of the optimal hyperplane in the form:

$$
w_{0} \sum_{i=1}^{N_{s v}} \alpha_{i} d_{i} x_{i}
$$

Summation is applying only to learning components for which the Lagrange multipliers are different from zero. They support vectors, the number of which is $N_{s v}$. Equation of the optimal hyperplane depends only on supporting vectors. Other vectors from the training set do not affect on the outcome of the decision. The equation defining the output $y(x)$ of the SVM linear network with optimal weights is expressed by the following relation:

$$
y(x)=\mathfrak{w}_{0} x+b=\sum_{i=1}^{N_{s v}} \alpha_{i} d_{i} x_{i}^{T} x+b
$$

This is a linear equation for the input variables described by the vector $x$ and scales that depend on the Lagrangian non-zero multipliers, their corresponding $N_{s v}$, support vectors xi and given values $d_{i}$.

Linear non-vaporization of patterns does not mean that they are not separated at all. As a rule, if $x$ is an input vector that describes a picture, then after resetting it into K-dimensional space, it is represented by a set of signs $\varphi_{j}(x)$ for $j=1,2, \ldots, K$. As a result of this 
transformation the hyperplane equation in a linear space is determined by the formula:

$$
g(x)=w^{T} \varphi(x)+b=\sum_{j=1}^{N} w_{j} \varphi_{j}(x)+b=0,
$$

where $w_{j}$ means the weights leading from $\varphi_{j}(x)$ to the output neuron, the weight vector $\mathrm{w}$ is $\mathrm{K}$-dimensional and $b$ is the weight of the polarization. Properties of the process described by the functions $\varphi_{j}(x)$ assumed the role of the individual variables $x_{j}$. The value of the output signal is described by the equation:

$$
y(x)=w^{T} \varphi(x)+b .
$$

The solution of primary problem is accomplished by transforming into a dual task, as well as in networks with linear separation of samples by minimizing the Lagrange function:

$$
\begin{gathered}
J(x, \mathcal{B} \& \text { \& } \mu)=\frac{1}{2} w^{T} w+ \\
+C \sum_{i=1}^{p}\left[d_{i}\left(w^{T} \varphi\left(x_{i}\right)+b-1+\epsilon_{i}\right] \alpha_{i}=\sum_{i=1}^{p} \mu_{i} \epsilon_{i} .\right.
\end{gathered}
$$

Solution of this optimization problem in the first step equals to assuming partial derivatives of the Lagrange function relative to $w, b$ and $\varepsilon$ to zero. The primary problem turns into a dual problem defined by the Lagrange multipliers $\alpha_{i}$ with the following form:

$$
\begin{gathered}
\max \left\{\sum_{i=1}^{p} \alpha_{i}-\frac{1}{2} \sum_{i=1}^{p} \sum_{j=1}^{p} \alpha_{i} \alpha_{j} d_{i} d_{j} K\left(x_{i}^{T} x_{j}\right)\right\}, \\
0 \leq \alpha_{i} \leq C, \quad \sum_{i=1}^{p} \alpha_{i} d_{i}=0 .
\end{gathered}
$$

The function $\mathrm{K}\left(x_{i}, x_{j}\right)$ in the formulated double problem is the scalar product of the vector function $\varphi(x)$ $=\left[\varphi_{I}(x), \varphi_{2}(x), \ldots, \varphi_{K}(x)\right] \mathrm{T}:$

$$
K\left(x_{i}^{T} x_{j}\right)=\varphi^{T}\left(x_{i}\right) \varphi\left(x_{j}\right) .
$$

This product defines the so-called kernel function.

Finally, an expression which specifies the output of the nonlinear SVM network can be written as:

$$
y(x)=w^{T} \varphi(x)+b=\sum_{i=1}^{N_{s v}} \alpha_{i} d_{i} K\left(x, x_{i}\right)+b,
$$

which depends on the kernel function $\mathrm{K}\left(x_{i}, x_{j}\right)$ and not from the activation function $\varphi(x)$.

Due to the classification problem, which requires a division of data into more classes, it is necessary to perform several classifications by using the methods "one against all" and "against each other". In the "one vs all" method in M classes are defined M SVM networks that determine exactly one class. The method requires preparation of M SVM, each of which learns on another set of data. After training all $\mathrm{M}$ networks there is a stage of reproduction, in which the same $x$ vector is fed into each SVM network and the output signals (functions of $\mathrm{M}$ decisions) of all trained SVM are determined. The SVM network was chosen to assign model classification through generalizing abilities. The SVM network is somewhat sensitive to selected hyperparameters that determine the number of neurons in the hidden layer.

The presented algorithm for classification of waste in the optical separator, based on the SVM network in the classical version, allows one to split fractional components on the basis of certain classes of standards described by the vector of parameters characterizing spectral bands, texture, structure and form. Due to the need to increase the quality control indicator of technological process carried out in an optical separator in the context of obtaining products with precisely defined physico-chemical, combustible and emission properties, the SVM algorithm was modified by extending the library of templates of primary classes (determined on the basis of spectra, textures and geometric characteristics) library of certain class standards, which are described by physico-chemical and inflammable properties (heat, humidity, content of volatile parts).

The parent algorithm, that is greedy approximation algorithm which solves the problem of covering a plurality, is responsible for the formation of class templates corresponding to optimized non-interlocked subsets of a set whose components are components of the waste fractions directed to the optical separator.

Reference classes are described by a set of attributes (texture, structure, spectrum) that allows lower level classification algorithm to be classified using the SVM network model (MC SVM) based on a set of functions intended for predefined classes represented by disjoint subsets of coverage by using Greedy Set Cover algorithm.

The modification of algorithm for solving the problem of optimization consists in realization of a method which allows one to check that a certain component (on the basis of image analysis) belongs to a class standard determined on the basis of fuel and physico-chemical properties and meets the criterion of the formulated target function, that is:

$$
\max _{u \in S} W_{d}^{T} u, \quad S=\left\{u \in R^{n}: A u \leq b_{\max }, A u \geq b_{\min }, u \geq 0\right\},
$$

where $W$ is the vector of the dimension $|j|$ (number of fractions components involved in the process of fuel component forming) with the components $w_{j}$, indicating the value of resulting fuel components heating. The process of optimization is reduced to the problem of linear programming (linear target function and linear constraints) with constraints imposed on the decision variables defined by the vector $U$, with the components $u_{j}$, which determines the set of admissible solutions:

$$
U=\left(u_{j}\right)=\left[u_{1}, u_{2}, \ldots u_{j}\right]^{T} .
$$

with dimension $|j|$ such $u_{j}$ means the share of $j$-th fuel fraction. 
The target function specified in the general form can be written as follows:

$$
W_{d}(u)=w_{d_{1}} u_{1}+w_{d_{2}} u_{2}+\ldots+w_{d_{j}} u_{j}=\sum_{j=1}^{n} w_{d_{j}} u_{j} \Rightarrow \max ,
$$

while the system of boundary equations in the form:

$$
\begin{gathered}
B_{\text {min }} \leq A U \leq B_{\max }, \\
B_{\text {min }}=\left(b_{k}^{\min }\right)=\left[b_{1}^{\min }, b_{2}^{\min }, \ldots b_{k}^{\min }\right]^{T},
\end{gathered}
$$

means a one-dimensional matrix (vector of left boundary equations) of dimension $|k|$ such that components mean the minimal participation of $b$ in fomation:

$$
\left(b_{k}^{\min }\right)=\left\{\begin{array}{l}
b_{1}^{\min }=\text { chlorine }(\mathrm{Cl}), \\
b_{2}^{\min }=\text { sulfur }(\mathrm{S}), \\
b_{3}^{\min }=\mathrm{PCB}, \\
b_{4}^{\min }=\text { metal }, \\
b_{5}^{\min }=\text { mercury }(\mathrm{Hg}), \\
b_{6}^{\min }=\text { cadmium }(\mathrm{Cd}),
\end{array}\right.
$$

while:

$$
B_{\max }=\left(b_{k}^{\max }\right)=\left[b_{1}^{\max }, b_{2}^{\max }, \ldots b_{k}^{\max }\right]^{T},
$$

means a one-dimensional matrix (vector of right sides of boundary equations) dimension $|k|$ such that components mean the maximum content of harmful substances. The values of the individual components of the vector are similar to $B_{\min }=\left(b_{k}^{\min }\right)$.

\section{Greedy Set Cover collection optimization problem solving method}

The method parameter is a pair $(\mathrm{X}, \mathrm{F})$ consisting of a finite set X (a set of waste fraction components) and a family of $\mathrm{F}$ subsets $\mathrm{X}$ (corresponding to predefined class standards determined on the basis of fuel, physicochemical properties and correspond to the criterion of the formulated target function, i.e. $\max _{u \in S} W_{d}^{T} u$, such that each element of the set $\mathrm{X}$ belongs to at least one member of the family F: $X=\cup_{S \in F} S$. In this case, the subset $S \in F$ covers its elements. The subfamily $\xi \subseteq F$, whose elements cover the whole set $\mathrm{X}:{ }^{X=\bigcup_{S \in \zeta} S}$, is the solution of the method.

The list below is a realization (in the pseudocode) of the Greedy Set Cover algorithm:

GreedySetCover(X, F):

$$
\text { 1. } U \leftarrow X
$$

2. $\xi \leftarrow \varnothing$;

3. while $U \neq \varnothing$ : 4.choose $S \in F$ which maximizes $|S \cap U|$;

5. $U \leftarrow U-S$;

6. $\xi \leftarrow \xi \cup\{S\}$.

In each phase, $U$ means a set of elements that are not known. The $\xi$ contains built lid. Line 4 is the stage at which a greedy solution is made, i.e. subset $S$ is selected. It covers as many elements as possible that are without covering (components of fractions). After selecting $S$, its elements are removed from $U$ and $S$ is added to $\xi$. When algorithm ends, the $\xi$ is a subframe of $F$ covering $X$.

\section{Data model}

A mathematical model for optimization of process of producing a light fraction (calorific value) consisting of separation (in an optical separator) of heavy non-metal components with high heating values $\mathrm{Wd}$, from a fraction of waste flow is represented by a matrix (column vector). $Z=(z j)$ corresponds to components that store indexes of the corresponding class templates generated by the greedy optimization algorithm. $P^{k}=\left(\mathrm{p}_{i, j}\right)$ with the components $p_{i, j}$ containing the $i$-th component (fraction) contained in the waste stream is sent to the optical separator into the reference class. The number of predefined classes is determined by the number found by the Greedy Set Cover algorithm, i.e. by greedy approximation of the given coverage with the optimal subsets $P^{k}$ which correspond to the criterion (14).

The algorithm that exceeds SVM checks in each iteration whether a specific component (based on the spectroscopic spectrum analysis)/fraction belongs to a class standard determined on the basis of fuel and physico-chemical properties and meets the criterion of the formulated target function.

Summing up, the algorithm implemented in the monitoring layer of SCADA control layer must reproduce the structures of predefined class templates Greedy Set Cover subsets containing the fraction components described by a set of attributes, including physical and chemical properties, fuel properties, $\mathrm{Wd}$, texture, spectroscopic spectrum, structure. I.e., each subgroup representing the class of fractional components that generates fuel is formed by the effect of optimization of greedy set covering algorithm in accordance with criteria for $\mathrm{Wd}$ and constraints on optimization problem solution variables [12-14].

\section{Component-oriented model}

In connection with the necessity of the managerial decisions sequence making problem solving (generating optimal trajectories of control decisions for the actuators of the optical separator), a polymorphic component model of a class was presented. It represents the algorithm of MS SVD, taking into account changes in its definition, with a logical representation of knowledge about an object for which the learning process is based 
on consistent verification, updating of knowledge and use of the results of this update to verify and reconstruct data structures.

The class type TMultiClassSupportVectorMachine contains the definition of a field with a logical representation of knowledge about management of technological process and methods of implementing algorithms that implement the tasks of specialization and adaptation of class component, in response to changing the state of equipment and technological parameters. Specialization follows the learning process in space of states and events by validating, reconstructing object data structures and reconfiguring control algae.

The component-oriented model of heavy screens separation in the optical separator is computer implementation of mathematical models (sets of data and algebraic expressions) describing individual components (objects, processes, relationships) [9-11]. The essence of the model definition is the use of special types of classes, structure of which allows one to display the specific properties of object components and to perform operations on data structures in the form of fields and methods. Object fields have another important advantage that allows one (via appropriate assignments) to refer to components created in other programs, such as Matlab, which run in different address spaces and provide a number of component categories stored in executable libraries (DLLs or EXE). They can be used to develop their own programs, directly using predefined interfaces or by defining child objects. The purpose of these links is to use the properties and methods (components of objects of predefined libraries).

The MC SVM algorithm "one vs all" can be written as follows:

Input: category $N, D=\left\{D_{1}, D_{2}, \ldots, D_{N}\right\}$ for training samples; testing sample $T$;

Output: Categories T;

Algorithm:

1.// educational section

2. for $n=1-N$ :

a. positive Sample $\leftarrow D_{N}$, negative Sample $\leftarrow$ other samples other than $D_{N}$;

b. save the data $\mathrm{SVM}_{n}$ classifier;

\section{3.//test section}

4. for $n=1-N$ :

a. use the $\mathrm{MSVM}_{n}$ classifier to calculate the value of $f(x)_{n}$;

5. compare all $f(x)_{n}$, output a maximum of $f(x)_{n}$.

\section{Results and discussion}

In order to verify numerical model and to validate its computer code, sample problems were simulated and the results were compared with the analytical solutions.

Validation of the model wass carried out with the following assumptions:
1. For the fuel formation process two components are used: dark coal P1 [kg i/kg] and combustible municipal

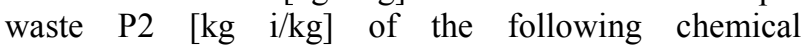
composition (c, s, h, o, n, w, p, cl):

P1: $(0,610 ; 0,003 ; 0,041 ; 0,0897 ; 0,0001 ; 0,0714 ; 0,1842$; $0,0074)$,

P2: $(0,5001 ; 0,0092 ; 0,0676 ; 0,2186 ; 0,1358 ; 0,02$; $0,0471 ; 0,0016)$

2. Optimisation constraints imposed on fuel formation process are used as given following: $\mathrm{s}<0,01$ and $\mathrm{cl}<0,003$

As a result of computer simulations and laboratory experiments the model gives the results that are very close to the obtained from the real process. Calculated Root Mean Square Error (RMSE) was as follows: a. for mass fraction of chlorine in formed fuel : $R M S E=0,00019(\mathrm{~kg} \mathrm{Cl} / \mathrm{kg})$

b. for mass fraction of sulphur in formed fuel : $R M S E=0,00038(\mathrm{~kg} \mathrm{~S} / \mathrm{kg})$

c. for $(W d)$ of formed fuel : $R M S E=47,81(\mathrm{~kJ} / \mathrm{kg})$

Application of the advanced algorithm for fuel formation process allowed the identification of the optimal mass fractions of components $\mathrm{P} 1$ and $\mathrm{P} 2$ in formed fuel (within the range of $0,01-0,24 \mathrm{~kg} \mathrm{i} / \mathrm{kg}$ ) and (within the range of: $0,76-0,99 \mathrm{~kg} \mathrm{i} / \mathrm{kg}$ ) with the constraints on the decision variables. Optimum value of the objective function $(W d)$ was found within the range of 22,86 to $25,12 \mathrm{MJ} / \mathrm{kg}$. The acceptable agreement between our simulation results and the measured data demonstrates that the model can reasonably simulate the real process of fuel production from separate combustible fractions of municipal wastes. The algorithm has been implemented in the SCADA system, in one of the companies producing fuels from waste. Energy savings of $15 \%$ per year have been achieved.

\section{Conclusions}

The integrated multithreaded process control algorithm, embedded in a programmable logical PLC controller (controlled by the SCADA system), performs the task of solving the optimization problem defined in the form of neural network model (Support Vector Machine) of nonmetallic fraction components, which reproduces the structures of predefined classes, i.e. subsets generated by the greedy algorithm of the Greedy Set Cover approximation containing fraction components described by a set of attributes (including physical and chemical properties, fuel properties, emissions, texture, structure and spectra).

Each subset, representing the model class of component fuel fractions, is generated by using the optimization effect of greedy algorithm of the Greedy Set Cover approximation, maximizing the purpose of the target (Wd-calorific value) when performing the technological constraints imposed on the solution of variable tasks linear programming. Reference classes are described by a set of attributes (texture, structure and spectrum) that allow the classification algorithm of lower level classification to be classified using the SVM network model (MC SVM) based on a set of functions 
intended for predefined classes represented by disjoint subsets of the Greedy Set Cover algorithm coverage and finally verify that the identified component/fraction belongs to the matrix of the class on the incident matrix.

\section{References}

1. S. Amponsah, S. Salhi, The investigation of a class of capacitated arc routing problems: the collection of garbage in developing countries, Waste Management, 24:711-721 (2004)

2. O. Apaydin, M. Gonullu, Route optimization for solid waste collection: Trabzon (Turkey) case study. Global NEST Journal, 9(1):6-11 (2007)

3. K. Barlishen, B. Baetz, Development of a Decision Support System for Municipal Solid Waste Management Systems Planning, Waste Management and Research, 14:71-86 (1996)

4. K. Gaska, A. Generowicz, I. Zimoch, J. Ciuła, Z. Iwanicka, A high-performance computing (HPC) based integrated multithreaded model predictive control (MPC) for water supply networks. Architecture Civil Engineering Environment, 10 (4):141-151 (2017)

5. K. Gaska, A. Generowicz, I. Zimoch, J. Ciuła, D. Siedlarz, A GIS based graph oriented algorithmic model for poly-optimization of waste management system. Architecture Civil Engineering Environment, 4:152-159 (2018)

6. K. Gaska, A. Generowicz, J. Ciuła, A HPC (High Performance Computing) based model for optimisation of the waste management systems in metropolitan regions. Technical Transactions 3:153163 (2018)

7. K. Gaska, K. Pikon, Methodology of biomass fuels creation processes; Twenty-sixth Annual International Conference on Incineration and Thermal Treatment Technologies - IT3'07 Conference, May 14-18, 2007, Phoenix, USA (ISBN: 9780923204822)
8. D. Kowalski, B. Kowalska, T. Bławucki, P. Suchorab, K. Gaska, Impact Assessment of Distribution Network Layout on the Reliability of Water Delivery. Water, 11:480 (2019), doi:10.3390/w11030480

9. A. Generowicz, K. Gaska, G. Hajduga, Multicriteria Analysis of the Waste Management System in a Metropolitan Area, E3S Web of Conferences, 10th Conference on Interdisciplinary Problems in Environmental Protection and Engineering. 2018, doi: 10.1051/e3sconf/20184400043

10. J. Ciuła, K. Gaska, A. Generowicz, G. Hajduga, Energy from landfill gas as an example of circular economy, E3S Web of Conferences, Vol. 30, The First Conference of the International Water Association IWA for Young Scientist in Poland "Water, Wastewater and Energy in Smart Cities", Cracow, Poland, 2018, doi: 10.1051/e3sconf/20183003002

11. K. Gaska, A. Generowicz, Advanced computational methods in component-oriented modelling of municipal solid waste incineration processes, Architecture Civil Engineering Environment, 10(1):117-130 (2017)

12. S. Werle, Impact of feedstock properties and operating conditions on sewage sludge gasification in a fixed bed gasifier, WASTE MANAGEMENT \& RESEARCH, 32(10):954-960 (2014), doi: $10.1177 / 0734242 X 14535654$

13. M. Smol, J. Kulczycka, A. Henclik, K. Gorazda, Z. Wzorek, The possible use of sewage sludge ash (SSA) in the construction industry as a way towards a circular economy. Journal of Cleaner Production, 95:45-54 (2015)

14. M. Smol, J. Kulczycka, Z. Kowlaski, Sewage sludge ash (SSA) from small and large incineration plants as a potential source of phosphorus - Polish case of study, Journal of Environmental Management, 184(3):617-628 (2016) 